\title{
Compétences interactionnelles et métier de service en restauration: les enjeux méthodologiques d'un corpus complexe
}

\section{Interactional skills and table waiting activity in restaurants: the methodological issues of a complex corpus}

\author{
Céline Alcade-Lebrun ${ }^{1, a}$ \\ ${ }^{1}$ LabEx Aslan, UMR ICAR (CNRS, Université Lyon 2, ENS de Lyon), École Normale Supérieure de Lyon - Site \\ Descartes, 15, Parvis René Descartes, BP 7000, 69342 LYON cedex 07 France
}

\begin{abstract}
Résumé. Notre contribution aborde la problématique méthodologique des choix effectués déterminant les modalités d'approche du terrain et de recueil de données. Dans un premier temps, nous replaçons notre étude dans le triple contexte, scientifique, éducatif, et professionnel, qui en a fait émerger son impérativité, ainsi que les partenaires qui en ont été à l'origine et les objectifs qui lui ont été assignés. Nous envisageons ensuite les contraintes et spécificités auxquelles nous avons été confrontée dans notre démarche de constitution de corpus pour mener à bien notre recherche, et développons les caractéristiques originales sur lesquelles est basé le dispositif de recueil de données hétérogènes adopté, en mettant en lumière les choix auxquels il a répondu, les questions éthiques et méthodologiques que ce dispositif a générées, et l'exploitation de données qu'il a permis de réaliser. Dans un troisième temps, nous explicitons de quelle manière, en plusieurs étapes successives et sur le long terme, le processus de production de données a fait évoluer notre questionnement de recherche dans la perspective de nos objectifs d'étude. Cette analyse nous permet finalement de considérer la question de l'articulation et du traitement des différents types de données en faveur d'une cohérence globale de notre étude.
\end{abstract}

\begin{abstract}
Our contribution addresses the methodological matters brought forward by the choices made in terms of field approach and data collection. In the first instance, we place our study back into the threefold context, - scientific, educational, and professional -, from which emerged an imperative need for it, along with the awareness of the partners that originally designed the research project, as well as the objectives assigned to it. Secondly, we consider the constraints and specificities we had to deal with during the constitution of our corpus in order to successfully achieve our research program. We examine the particular features that characterize the methodology we opted for with the aim of collecting data from different sources, as well as the choices on which it is based, the ethical and methodological issues it raised, and the particular processing it permitted us to complete. Thirdly and finally, we clarify how, during several successive steps in a long-term perspective, the data production process evolved our research questions in regard to the initial objectives of our study. This analysis ultimately enables us to contemplate the question of articulating and processing heterogeneous data generated by diverse sources in order to ensure overall coherence of our research.
\end{abstract}

\footnotetext{
${ }^{a}$ Auteur de correspondance : celine_alcade@yahoo.fr
}

This is an Open Access article distributed under the terms of the Creative Commons Attribution License 4.0, which permits unrestricted use, distribution, and reproduction in any medium, provided the original work is properly cited. 


\section{Cadre général de la recherche}

Des enjeux convergents dans différents domaines ont conduit à initier l'étude que nous présentons. Dans le champ scientifique tout d'abord, une recherche récente (Hugol-Gential, 2012 [1]) a démontré l'impact déterminant des interactions clientèle-personnel de service sur l'appréciation globale des repas au restaurant. Or, paradoxalement, dans le domaine de la formation, l'examen des programmes de différents types d'établissement français préparant à acquérir les compétences professionnelles du service en restauration (CFA, Lycée hôtelier, Instituts supérieurs) révèle que cet aspect langagier et interactionnel ne fait pourtant pas explicitement partie des enseignements de base. Enfin, concernant l'orientation des jeunes vers les métiers de bouche, l'A.N.P.C.R. (Association Nationale des Professeurs de Cuisine et Restaurant des C.F.A.) met en avant une nette tendance à la dévalorisation des métiers de salle (qui ne représentent que $25 \%$ des choix de carrière), au profit des métiers de cuisine (rassemblant $75 \%$ des vocations), alors que les deux filières présentaient un équilibre vingt ans auparavant.

Sensibles à ces enjeux, l'Université Lyon 2 via le Laboratoire ICAR et le LabEx Aslan, ainsi que L'Institut Paul Bocuse (Ecully, France), établissement de formation privé spécialisé dans le secteur de l'hôtellerie-restauration, ont noué un partenariat afin de réaliser cette recherche. Au croisement des sciences du langage et des sciences de l'éducation, elle a été axée sur deux objectifs spécifiques. Premièrement, un objectif scientifique vise la documentation des pratiques de formation. Il consiste à étudier les compétences langagières et interactionnelles en jeu au cours de l'activité de service au restaurant, ainsi que leurs processus de transmission. Deuxièmement, un objectif opérationnel ambitionne de formuler et expérimenter de nouvelles pratiques didactiques de formation aux compétences évoquées afin d'améliorer leur transmission dans une logique d'ingénierie didactique collaborative menée avec les formateurs de l'institution.

Pour aborder ces axes de recherche, sur un plan théorique, notre étude s'inscrit dans la continuité des recherches qui font le lien entre la didactique professionnelle (Kunégel, 2011 ; Mayen, 2005 ; Pastré et al., 2006 [2-4]), les workplace studies (Billett, 2001 ; Lave et Wenger, 1991 [5,6]), et la linguistique interactionnelle (Goffman, 1967, 1973, 1974 ; Gumperz, 1989 ; Hymes, 1984 ; Mondada, 2006 ; Searle, 1972 ; Vygotsky, 1985 [7-14]) et multimodale (Filliettaz et al., 2008 ; Kress et al., 2001 $[15,16])$, replaçant le langage dans une théorie de l'action. Nous adoptons, dans cette logique, une démarche interdisciplinaire, à la croisée de ces différents champs, auxquels nous ajoutons celui de la sociolinguistique (Borzeix et Gardin 1992 ; Boutet, 2001 [17, 18]), qui nous paraît pertinente pour replacer la formation dans le cadre dans lequel elle intervient.

\section{Approche méthodologique et dispositif de production de données}

Le choix des modes de production de données devant constituer notre corpus découle de façon cohérente des objectifs de recherche initialement identifiés et des hypothèses formulées en vue de les atteindre de la façon la plus satisfaisante possible. Néanmoins, il résulte également des contraintes et spécificités propres à notre terrain, l'Institut Paul Bocuse. Elles se rapportent à la structuration intrinsèque de la formation au métier de service en hôtellerie-restauration que cet institut a mise en place, ainsi qu'aux conditions d'accès qu'il nous a offertes pour l'étudier. Ces éléments ont déterminé les stratégies, tant sur le plan éthique que méthodologique, que nous avons adoptées pour aborder nos observations.

Notre terrain d'étude a été rapidement circonscrit à la première année de la Licence professionnelle en Management de l'Hôtellerie-Restauration (Filière HR) proposée au sein de l'Institut Paul Bocuse, car elle constitue une année privilégiée spécifiquement dédiée au travail des compétences visées.

Si l'accès à ce terrain ne s'est aucunement avéré problématique en raison des vives sollicitations de la direction dont notre recherche a fait l'objet, nous n'en avons pas moins été confrontée à une quête nécessaire de légitimité auprès des maîtres d'hôtel-formateurs. Cet enjeu s'avérait d'autant plus important que ces formateurs constituent le sésame d'accès au terrain de la formation à la pratique du 
service en restauration proposée par l'Institut Paul Bocuse. Elle se déroule au sein de ses trois restaurants-écoles, positionnés sur des offres de restauration différentes : le $F \& B$, remplissant le rôle de cantine haut de gamme du personnel de l'Institut ; L'Institut, faisant office de brasserie de luxe, et Saisons, le restaurant gastronomique de l'établissement. Effectivement, en tant que responsables des trois semaines d'activité de service en salle suivies par les étudiants de première année, ce sont ces personnes qui se trouvent principalement en charge de transmettre les compétences langagières et interactionnelles au fondement de l'expertise du métier sur lesquelles nous nous focalisons. Par compétences langagières et interactionnelles, nous nous référons à la notion de compétence en tant que savoirs d'action telle que l'évoque Filliettaz (2008, [15]) et les définissons par le phénomène même de mobilisation des ressources langagières (verbales et non verbales), à la fois individuelles et collectives, qui fondent l'expertise du métier de service en permettant de réaliser de façon performante les actions qui en découlent (prendre la commande, proposer, conseiller, présenter les plats, etc.). Nous nous sommes finalement vu accorder cette légitimité attendue par les maîtres d'hôtel-formateurs par le fait de suivre, sans caméra et sur la base d'un journal de terrain, une formation au service en restauration au même titre qu'une étudiante de première année, avant de commencer nos observations proprement dites, pour laquelle nous avons eu recours à l'utilisation d'enregistrements audio-vidéo. Cette formation s'est avérée cruciale, non seulement pour connaître de "l'intérieur » et de façon pratique les enjeux et réalités du métier, mais également afin de pouvoir véritablement intégrer l'équipe pédagogique, après avoir fait nos preuves sur le terrain des restaurants-écoles.

Nous sommes ensuite revenue sur le terrain de la formation en restaurant-école, non plus cette fois avec le statut d'étudiante, mais de collaboratrice engagée dans un travail d'ingénierie de la formation, en partenariat avec les maîtres d'hôtel-formateurs, destiné à, nous le rappelons, formuler et expérimenter de nouvelles pratiques didactiques de formation aux compétences évoquées afin d'améliorer leur transmission. Concernant le choix du dispositif filmique alors adopté pour ces nouvelles observations, les caractéristiques spatiales et organisationnelles des restaurants-écoles, ainsi que les aspects de la formation pratique à étudier ont constitué des contraintes importantes. Nous avons opté pour un dispositif mobile (Veillard, 2013 [19]), consistant en une caméra à l'épaule légère et maniable, sans micro additionnel. Les raisons de ce choix sont multiples et nous semblent largement compenser les inconvénients qu'il génère, qui résident dans la qualité relativement moyenne de l'image et du son, ainsi que l'absence de multiplicité de points de vue d'un même phénomène. Elles concernent tout d'abord la nécessité de respecter au mieux l'écologie des espaces de formation et production de services que sont les restaurants-écoles, qui souffriraient d'un matériel de prise de vue trop invasif. D'une part, cela porterait atteinte à l'intimité liée à la perception de l'espace, au cours des échanges entre clientèle, personnel de service et étudiants. D'autre part, de nombreuses personnes circulant entre les divers lieux qui composent les restaurants-écoles (office, cuisine, salle(s) de restaurant), au cours d'une activité corrélée à des rythmes relativement soutenus, il était impératif qu'elles ne soient pas entravées dans leurs déplacements. De plus, il s'avérait indispensable de laisser un rôle actif et une latitude relativement grande à l'observateur sur le terrain, quant aux choix possibles de cadrages, de plans variables et rapidement ajustables, en fonction des situations et pratiques étudiées (Lallier, 2009 [20]). Enfin, nous avons choisi de programmer un temps d'observation sur de relativement longues périodes (un mois et demi la première année, trois mois la seconde), dans le but de pouvoir notamment étudier les évolutions des pratiques des acteurs, ce qui ne peut se faire que sur le long terme. Aussi, la nécessité d'une économie de matériel de captation, tant en termes d'installation que de temps de traitement des données, ont également orienté le choix d'un tel dispositif.

Afin d'éclairer le positionnement d'observatrice pour lequel nous avons opté, et qui a résulté à la fois d'un choix stratégique et des contraintes d'accès au terrain, nous aimerions revenir à la façon dont nous avons intégré l'équipe de service. Cette dernière est composée du maître d'hôtel formateur et d'un groupe de 6 à 10 étudiants, ainsi que, selon les restaurants-écoles, d'un(e) assistant(e), voire de serveurs professionnels supplémentaires. Concernant notre intégration dans cette équipe, le fait d'avoir suivi, comme évoqué précédemment, une formation au métier, et d'en connaître les aspects et processus essentiels, nous a positionnée aux yeux des maîtres d'hôtel-formateurs comme une 
personne-ressource, à la fois pour les seconder dans leur dynamique de formation auprès des étudiants, mais également afin d'anticiper et pallier les impératifs de l'activité lors de son déroulement en cas de non disponibilité de leur part. Dans un premier temps, nous avons douté de pouvoir tirer avantage d'un tel positionnement, et nous avons manifesté une certaine résistance auprès des formateurs à l'adopter. Mais les bénéfices furent tels que nous avons été rapidement convaincue de l'intérêt de cette posture d'observatrice participante. En effet, assumer une telle position nous permettait, d'une part, de suivre de façon extrêmement précise et attentive les contingences de l'instant, de même que les actions et interactions de participants, en se trouvant au plus près de l'activité et de ses protagonistes. Elle nous assurait ainsi de demeurer dans l'anticipation des tâches devant être mises en œuvre, et donc, par là-même, extrêmement vigilante aux situations importantes à observer. D'autre part, cette coopération dans le travail nous a apporté la confiance des formateurs et étudiants, qui ont dès lors perçu notre présence comme bienveillante puisque impliquée, et utile, étant donné que, premièrement, nous convergions vers leurs impératifs de travail, et deuxièmement, pouvions leur apporter des outils leur permettant d'améliorer leurs pratiques. De plus, étant sensibilisée aux enjeux de l'activité, les ayant adoptés, et disposant des connaissances pour pouvoir contribuer aux actions de formation et de service, il aurait été extrêmement difficile, et au vu des éléments que nous venons d'exposer, préjudiciable pour notre étude de nous positionner autrement. Finalement, les différents acteurs des situations observées ont assez rapidement intégré ce choix, en percevant la caméra comme un instrument faisant entièrement partie de notre activité de production de service, recherche en collaboration, et formation, devenue conjointe à la leur. Vis-à-vis de l'analyse des données, le fait que la caméra soit, par le choix du dispositif de prise de vue, assimilée à notre regard, nous a permis d'en expliciter à la fois le cadrage, comme le hors-cadre, du fait d'avoir mémorisé les circonstances vécues des situations filmées (retranscrites dans notre journal de terrain).

Cependant, la production d'enregistrements filmiques ne pouvait suffire pour atteindre nos objectifs de recherche. En effet, il ne s'agissait pas seulement de documenter visuellement les actions et interactions situées au sein des restaurants-écoles de l'Institut Paul Bocuse, mais également de disposer de sources d'information complémentaires, indispensables pour circonscrire, analyser et interpréter dans leur globalité les pratiques de transmission-apprentissage des compétences langagières et interactionnelles. Par exemple, il fallait pouvoir étudier en quoi certains cours « théoriques» apportaient des savoirs utiles pour nourrir les échanges langagiers avec les clients et les autres professionnels des restaurants (par exemple, un cours sur la perception et description sensorielle des aliments ; ou un autre sur les vins, fait par un sommelier, etc.). De ce point de vue, l'absence de référentiel formalisant a priori les différents contenus d'enseignement de la filière, ainsi qu'un certain manque de coordination et d'information mutuelle au sein de l'équipe pédagogique, ont notamment constitué des difficultés propres à l'institution choisie. Elles nous ont conduite à étudier scrupuleusement l'ensemble des modalités de cours, établies en fiches descriptives individuelles (syllabus) rédigées par chaque formateur, afin de connaître la teneur précise des contenus enseignés, confirmer leur pertinence par rapport à la transmission des compétences étudiées, et, dans ce cas, les intégrer à nos observations. Dans ce but, lorsque la description des fiches s'avérait trop imprécise, il a parfois été nécessaire de compléter cette approche par des entretiens auprès des formateurs concernés. Nous nous sommes également ensuite concertés avec le responsable des formateurs chargés de l'enseignement en restaurants-écoles et la responsable pédagogique de la filière HôtellerieRestauration afin qu'ils cautionnent la validité de notre sélection. Il s'agissait également de pouvoir situer nos observations dans un certain contexte professionnel, où les différents acteurs et institutions développent des discours sur ce que doit être le service en salle, sa légitimité. Nous souhaitions donc disposer d'autres sources pour connaître certaines représentations du métier et stratégies développées par les formateurs auprès des étudiants.

Ces objectifs d'étude, suivant une approche praxéologique et multimodale de l'enseignementapprentissage étudié (Filliettaz, De Saint-Georges, Duc, 2008 [15]), ont par conséquent impacté notre choix méthodologique concernant le recueil de données. Pour y répondre, nous avons opté pour une démarche relevant de l'ethnographie de la communication (Gumperz et Hymes, 1964 [21]), basée sur l'enquête de terrain. Ce type d'enquête conduit à une combinaison complexe et originale de différents 
modes de production de données relativement hétérogènes, mais complémentaires (Baude et al., 2006 [22]). En référence à Olivier de Sardan (1995 [23]), nous les classons en quatre types, - observation, entretien, procédés de recension, collecte de documents écrits - (Cf. Tableau 1 des caractéristiques du corpus de données ci-dessous).

Dans notre étude, le mode de production de données qui s'est avéré le plus important en termes de volume et d'impact, a consisté dans la mise en place d'observations participantes des pratiques de formation. Nous les avons menées, non seulement lors des cours pratiques dans les restaurants-écoles, mais également lors de certains cours théoriques, pour la raison évoquée ci-dessus. Ces observations se déclinent sous la forme de prises de notes (journal de terrain), d'enregistrements audio et/ou vidéo, et de photos. Nous les avons complétées par des entretiens (enregistrements audio pour la plupart d'entre eux, ou notes sur notre journal de terrain lorsqu'ils étaient plus informels) avec les acteurs de la formation (formateurs et étudiants), ainsi qu'avec des experts du terrain de la restauration (service en salle et formation). Nous avons également mis en place pour la constitution de notre corpus, des procédés de recension systématique de données nous informant sur la structuration de la formation et son évolution. La collecte de documents écrits encadrant la formation constitue la dernière composante de notre corpus et concerne des documents pédagogiques (sur lesquels sont basés les enseignements) et institutionnels (retraçant l'historique de l'établissement, syllabus de cours, emplois du temps et répartition horaire des cours, etc.).

Tableau 1. Tableau récapitulatif des caractéristiques du corpus de données.

\begin{tabular}{|c|c|c|c|c|c|}
\hline \multicolumn{2}{|c|}{$\begin{array}{l}\text { Dispositif de } \\
\text { l'enquête de terrain }\end{array}$} & \multirow{2}{*}{$\begin{array}{l}\text { Informations révélées } \\
\text { par ces données }\end{array}$} & \multirow{2}{*}{\begin{tabular}{|l|} 
Acteurs impliqués \\
$\begin{array}{l}\text { Acteurs de la formation } \\
\text { (étudiants/formateurs) }\end{array}$
\end{tabular}} & \multirow{2}{*}{\begin{tabular}{|l|} 
Formats \\
Vidéo \\
Journal de terrain \\
(prise de notes)
\end{tabular}} & \multirow{2}{*}{$\begin{array}{l}\begin{array}{l}\text { Volume des } \\
\text { données }\end{array} \\
45 \mathrm{~h}(60 \mathrm{Go})\end{array}$} \\
\hline 1 & $\begin{array}{l}\text { Observation } \\
\text { participante }\end{array}$ & & & & \\
\hline 2 & Entretiens & $\begin{array}{l}\text { Discours et } \\
\text { représentations qui } \\
\text { sous-tendent la culture } \\
\text { des acteurs du terrain }\end{array}$ & $\begin{array}{l}\text { Acteurs de la formation } \\
\text { (étudiants/formateurs) } \\
\text { Experts de la profession }\end{array}$ & $\begin{array}{l}\text { Audio (formel) } \\
\text { Journal de terrain } \\
\text { (échange informel) }\end{array}$ & $9 \mathrm{~h}(600 \mathrm{Mo})$ \\
\hline 3 & $\begin{array}{l}\text { Procédés de } \\
\text { recension } \\
\text { systématique }\end{array}$ & $\begin{array}{l}\text { Évolution et variation } \\
\text { des modes de } \\
\text { structuration de la } \\
\text { formation }\end{array}$ & $\begin{array}{l}\text { Coordinateurs } \\
\text { pédagogiques }\end{array}$ & Contact mail/oral & $10 \mathrm{Mo}$ \\
\hline 4 & $\begin{array}{l}\text { Collecte } \\
\text { sources écrites }\end{array}$ & $\begin{array}{l}\text { Modes de } \\
\text { structuration des } \\
\text { pratiques et dispo- } \\
\text { sitifs encadrant la } \\
\text { formation }\end{array}$ & $\begin{array}{l}\text { Formateurs et coordina- } \\
\text { teurs pédagogiques } \\
\text { Directeurs académiques } \\
\text { Chargés de communication }\end{array}$ & $\begin{array}{l}\text { Documents } \\
\text { papier /numériques }\end{array}$ & $30 \mathrm{Mo}$ \\
\hline
\end{tabular}

\section{Production de données et évolution du questionnement}

En observant la figure ci-dessous (Fig. I) qui retrace les étapes successives de la production de données, s'étendant sur deux années de recherche, nous remarquons que ce processus a, au fur-et-àmesure des données produites, fait évoluer le questionnement à la base de notre étude, de manière imbriquée, à la fois à travers notre travail de recherche et d'ingénierie de la formation. 


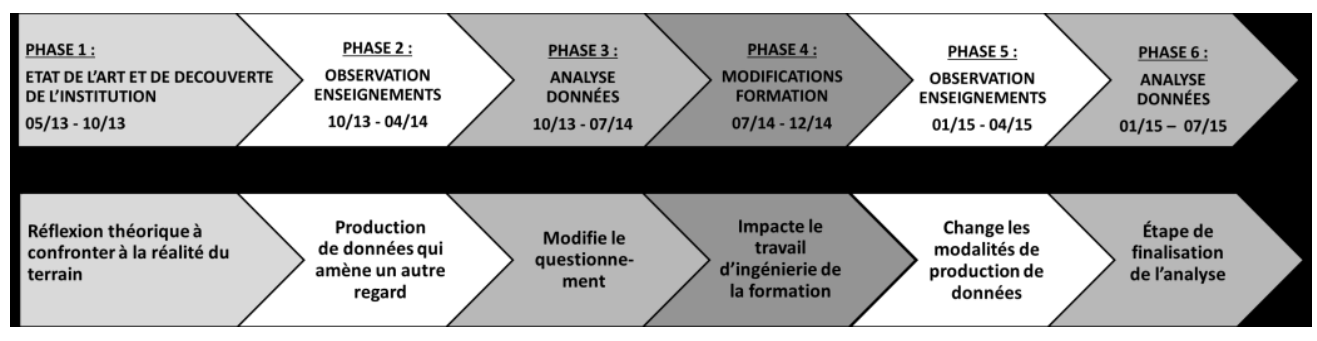

Figure 1. Évolutions parallèles de la production de données et du questionnement de recherche.

Ce cheminement réciproque qui s'est opéré entre production de données et questionnement de recherche, s'est effectivement déroulé de manière progressive. La première phase de travail, de mai 2013 à octobre 2014, a été dédiée à l'état de l'art de la recherche et à la découverte de l'institution, dans son organisation générale, et concernant la nature et structuration des enseignements de la première année de Licence professionnelle en Management de l'hôtellerie-restauration. À ce titre, une première série de données relevant des quatre composantes décrites a été générée.

Elle a été complétée par une phase consécutive d'observation participante des enseignements de la filière qui a suivi d'octobre 2013 à Avril 2014. Il s'est d'abord agi de l'observation (non enregistrée) des enseignements théoriques possédant un lien potentiel avec la pratique professionnelle du service en restauration. Ensuite, nous avons observé les enseignements pratiques en restaurants-écoles, en deux temps comme précédemment évoqué, avec premièrement une observation participante de la formation au métier de service avec le statut de simple étudiante (non enregistrée), suivie d'une observation participante en tant qu'observatrice-collaboratrice (avec recours à la caméra).

Cette deuxième étape a donné lieu à un travail d'analyse (troisième phase de travail), commencé en parallèle de ce temps d'observation, qui a permis de confronter le questionnement initial théorique à la réalité du terrain, en mettant notamment à jour des présupposés erronés antérieurs à l'observation (et sous-tendant son organisation) dont nous n'avions pas conscience. Premièrement, nous postulions que la transmission s'opérait nécessairement au cours de la formation sous la forme de relations bilatérales entre maître d'hôtel-formateur responsable de l'enseignement et étudiants), et qu'elles devaient constituer le focus de nos observations. Deuxièmement, nous supposions que les savoirs théoriques relatifs à l'activité de service en restauration, étaient nécessairement présentés aux étudiants en lien direct avec la finalité professionnelle de cette pratique professionnelle. Troisièmement, nous étions convaincue que les compétences langagières et interactionnelles, étant inhérentes à l'activité de service, faisaient déjà l'objet d'une identification complète, et, dans une certaine mesure, d'une transmission et d'un entraînement. Or, l'analyse du terrain a démenti ces présupposés en mettant en évidence les trois éléments suivants importants dans les pratiques de formation : tout d'abord des relations de transmission multilatérales entre maître d'hôtel-formateur, chef, clients, et étudiants dans une dynamique de co-construction des apprentissages (Vygotsky, 1985 [14]) ; ensuite un manque de transfert des connaissances transmises en amont, relativement peu mobilisées dans la pratique du service, et qui révèle un fort cloisonnement des enseignements; et enfin l'absence d'un travail de définition et d'entraînement à l'acquisition des compétences interactionnelles autres que la maîtrise des gestes techniques et procédures de service, sur le terrain de formation des restaurants-écoles.

En conséquence, ce travail d'analyse a abouti en juillet 2014 à une quatrième phase de propositions de complémentation et de modification de la formation dans une perspective d'amélioration de cette dernière, en réponse aux problèmes observés. Ces propositions ont pris en compte la multilatéralité des interactions de formation attestée sur le terrain, et visé à encourager la coopération et les échanges collaboratifs entre les différents acteurs concernés par l'activité de service en restauration, notamment en suggérant de faire intervenir le chef pour faire part aux étudiants de son approche et ses connaissances culinaires concernant la préparation des produits et des plats de sa carte. Les modifications proposées ont également consisté à favoriser la circulation transversale d'outils et savoirs pouvant bénéficier à divers enseignements et engager un travail de création de matériel 
pédagogique, cours et exercices, visant à combler les lacunes identifiées de la formation. Dans ce but, nous avons notamment suggéré de mettre en place dans chaque restaurant-école des séances de vidéo formation basée sur le visionnage d'extraits de prises de vues réalisées pendant le service. Ces séances consistent, à partir d'exercices d'auto-confrontation collective et d'observation de pairs (révélant stratégies et positionnement dans l'interaction), à assurer un entraînement à l'identification des compétences langagières et interactionnelles à la base de l'expertise du métier de service. Ces compétences sont mises en œuvre dans des phases-clés de l'activité de service (accueil du client, distribution des cartes, prise de commande, service des plats, des fromages et des desserts), et nous les caractérisons en termes de plus-value apportée par l'information, le conseil, et l'accompagnement du client avec assurance et sens de la théâtralité. Par le biais de ces séances, nous proposions donc d'identifier ensemble les raisons à l'origine d'interactions satisfaisantes ou non entre personnel de service et clients, au cours des différentes phases envisagées. Enfin, l'application des modifications susmentionnées, validées par la direction, a été menée en collaboration avec les formateurs de juillet à décembre 2014.

Ensuite, l'impératif de mesurer l'impact de ces modifications sur la transmission des compétences langagières et interactionnelles visées, a fait l'objet d'un retour sur le terrain de la pratique en restaurant école pour une deuxième phase d'observation (qui constitue une cinquième phase de travail). Lors de cette étape, les modalités de production de données initialement choisies pour la première phase d'observation ont également évolué. Elles ont été influencées par les phases précédentes, d'analyse des pratiques de formation au service en restauration et de collaboration avec les formateurs, qui avaient pour objectif de gagner en efficacité et cohérence dans nos finalités de transmission des compétences langagières et interactionnelles. Ces phases ont en effet révélé, qu'à travers l'acquisition de ces compétences communicationnelles, se jouait un phénomène de construction de l'identité professionnelle au fondement de l'expertise du métier de service. En conséquence, concernant l'observation des interactions de formation, nous avons opéré un changement de focus de prise de vue avec un élargissement des "couples interactionnels » visés : non seulement le couple maître d'hôtel-formateur/étudiants, mais également chef/étudiants, étudiants/clients, et étudiants entre eux. Suivant la même logique, l'étude de la seule performance de l'étudiant a été replacée non seulement dans le cadre de l'échange interactionnel avec la clientèle, mais également dans le cadre de la communication avec les équipes de service en salle et de cuisine, dont la dynamique engage l'efficacité du déroulement de l'activité professionnelle. Également, nous avons ajouté l'observation des interactions maître d'hôtel-formateur/clients, parce qu'elles nous sont apparues dans l'analyse comme contribuant de façon capitale à notre travail de définition et de construction des compétences langagières et interactionnelles à la base de l'identité et expertise professionnelles. De plus, pour répondre aux besoins de l'analyse de ces compétences, les événements de communication considérés, initialement focalisés sur le service et l'annonce des plats, ont eux aussi été élargis aux diverses phases de prises de commande, ainsi qu'à l'ouverture et la clôture des interactions avec la clientèle, que nous avons alors également envisagées comme des moments-clés mettant en jeu les compétences langagières et interactionnelles fondant l'expertise du métier.

La sixième et dernière phase de travail de terrain concerne la finalisation de l'analyse des données avant le processus même de rédaction de la recherche (qui constitue également une prise de recul et une maturation supplémentaires). Pour lors, cette analyse terminale a montré que certains formateurs impliqués dans la transmission de savoirs avant tout théoriques, avaient modifié à leur initiative leur contenu de cours pour l'axer davantage vers la finalité de la pratique professionnelle en restaurantsécoles. De plus les enseignants ont montré de l'intérêt à s'inspirer et s'emparer d'outils qu'ils ont découverts au cours de ce travail, et dont certains avaient été précédemment mis en place par d'autres formateurs. Enfin, nous avons remarqué sur le terrain qu'une posture communicationnelle nouvelle de la part des étudiants, basée sur l'information, le conseil et l'accompagnement en face-à-face avec la clientèle, avait émergé, pour faire enfin apparaître une certaine expertise langagière et interactionnelle garantissant l'assise d'une véritable identité professionnelle.

Ces remarques sur les processus conjoints de production de données et évolution du questionnement de recherche montrent clairement à quel point notre travail d'ingénierie de la 
formation nourrit notre travail scientifique et inversement, nous amenant finalement à considérer, dans un souci de cohérence globale dans notre approche des phénomènes étudiés, la question de l'articulation et du traitement sur le long terme de données hétérogènes.

Concernant notre choix méthodologique d'opter pour l'articulation de données hétérogènes dans le cadre de l'enquête de terrain, comme le souligne Olivier de Sardan (1995, [23]) il permet de rendre compte de manière la plus fine possible des «multiples registres et stratifications du réel » et servir ainsi au mieux nos objectifs de recherche. En référence à cet auteur, nous avons en effet d'abord opéré par triangulation complexe, procédé qui consiste à confronter les diverses données collectées, à recouper les informations de natures variées qu'elles révèlent ( $\underline{\mathrm{Cf}}$. Tableau 1 des caractéristiques du corpus de données), et qui justifient notre recours au mode de production respectif dont elles relèvent, car elles permettent d'expliciter les postures des acteurs dont elles proviennent, éclairant diversement et de façon complémentaire les phénomènes étudiés, permettant par conséquence de mieux les cerner. Également, nous avons procédé par itération abstraite, en effectuant, comme nous l'avons montré précédemment dans le processus de travail décrit, des allers-retours entre production de données et problématique de la recherche, résultats et interprétation, dans une dynamique d'influence et de modification réciproques qui a caractérisé le cheminement particulier de notre recherche.

Sur le plan du traitement du corpus constitué, nous avons suivi un processus de réduction par stratégie de raffinement progressif d'hypothèses (Veillard [19] en référence à Engle et al., 2007 [24]). Cette démarche a consisté à tout d'abord sélectionner dans chacune des quatre composantes de notre corpus (observations participantes, entretiens, documents institutionnels et documents pédagogiques encadrant la formation), les extraits pertinents en rapport aux dynamiques de transmission et de construction des compétences langagières et interactionnelles étudiées. Puis, nous nous sommes appliquée, en recourant au logiciel TRANSANA en ce qui concerne les extraits audio et vidéo, à identifier, décrire et caractériser de façon fine les composantes de ces phénomènes, au moyen des catégories conceptuelles relatives aux événements de communication théorisées par Hymes (1984, [11]), et permettant d'analyser les interactions de service comme de formation. Cette analyse nous a notamment conduite à mettre en lumière une dynamique collective positive de co-construction de la formation (Vygotsky, 1985 [14]), à identifier des problèmes de transfert et réinvestissement des connaissances vues en amont de la pratique en restaurants-écoles, et des problèmes de définition d'une identité professionnelle à transmettre et travailler dans la formation à travers les interactions avec la clientèle. Ensuite, nous avons recherché dans les différentes composantes de notre corpus, d'une part les facteurs pouvant expliquer les problèmes de transmission observés que nous venons d'évoquer, et, d'autre part, réfléchi aux éléments qui permettraient non seulement de tirer le meilleur bénéfice possible des ressources et des dynamiques individuelles et collectives présentes dans la formation, mais également de combler les lacunes de la formation (notamment en termes de travail et définition d'une identité professionnelle liée aux compétences langagières et interactionnelles). Dans le but de répondre à ces questions, nous avons été conduite à formuler un certain nombre d'hypothèses théoriques sur lesquelles nous nous sommes par la suite appuyée pour élaborer nos propositions de modifications de la formation. En ce qui concerne les problèmes soulevés de transfert et réinvestissement des connaissances, nous en avons déduit après analyse des données relatives aux cours théoriques (documents fournis en classe et connaissances transmises recensées dans notre journal de terrain), qu'ils étaient dus au cloisonnement des enseignements, à l'origine de la noncirculation entre cours théoriques et pratiques du matériel pédagogique utilisé ainsi que des savoirs transmis, dans un contexte peu coopératif au sein de l'équipe pédagogique. À ce sujet, nous notons qu'étudiants comme formateurs s'avèrent responsables de cette absence de remobilisation des connaissances constatée dans les divers enseignements théoriques et pratiques liés par la thématique professionnelle du service en restauration. Les problèmes de définition d'une identité professionnelle à transmettre aux étudiants basée sur des compétences langagières et interactionnelles, nous sont apparus quant à eux, après analyse des données vidéo collectées durant les observations participantes de séquences de formation et de services sur le terrain des restaurants-écoles ainsi que des données audio issues des entretiens avec les maîtres d'hôtel-formateurs, comme résultant du fait que ces enseignements pratiques envisagent de manière générale les seules transmission et maîtrise des 
procédures et gestes techniques, évacuant le travail du langage et de la communication qui pourtant contribuent également à asseoir l'expertise du métier. Nous avons alors rapporté ce phénomène à la difficulté rencontrée par les maîtres d'hôtel-formateurs (perceptible dans les entretiens et séquences de formation) à mentionner, définir et convoquer dans leur discours les compétences langagières qui fonde leur professionnalisme. De manière générale, nous constatons que celles-ci ne sont ni clairement identifiées, ni travaillées dans les cursus de formation aux métiers de l'hôtellerierestauration, à la faveur exclusive des procédures et gestes techniques. Aussi, les maîtres d'hôtelformateurs, lorsqu'ils évoquent ces compétences dans leurs propos, le font de façon imprécise, comme d'un élément qui s'acquiert "sur le tas », de manière intuitive, par expérience et observation de pairs. Par conséquent, n'étant pas explicitement abordée lors de la formation, ni visée comme un objectif à atteindre, cette notion d'identité professionnelle liée à la maîtrise du langage et de la communication, reste pour les étudiants un objet relativement peu identifiable, qui n'apparaît qu'à travers le modèle du maître d'hôtel-formateur, sous forme donc de compétence cachée ou tout du moins relativement invisibilisée. Enfin, une deuxième série d'observations participantes sur le terrain ayant pour but d'évaluer l'impact des modifications apportées à la formation sur l'acquisition des compétences langagières et interactionnelles visées, a produit de nouvelles données (enregistrements vidéos, entretiens et journal de terrain concernant les enseignements théoriques et pratiques liés à la formation au métier de service en restauration), dont l'analyse nous a permis d'affiner nos hypothèses ainsi que notre modèle théorique, et tester leur possible généralisation.

\section{Conclusion et perspectives}

Notre étude, a été basée sur l'enquête de terrain et, par conséquent, sur la mise en place d'un dispositif de production de données hétérogènes sur le long terme, composé d'observations participantes, entretiens, de procédés de recension systématique, et de collecte de sources écrites. Cette méthodologie nous a conduite à rassembler des matériaux aussi divers que des documents écrits (pédagogiques, institutionnels, extraits de journal de terrain), audio et/ou vidéo, posant le problème de l'articulation et du traitement des données.

Pour y répondre, en ce qui concerne l'articulation des données nous avons opéré par triangulation complexe et itération abstraite, (Olivier de Sardan, 1995 [23]). De cette manière, nous avons, d'une part, pu caractériser de manière extrêmement complète les pratiques de formation étudiées, par la confrontation permanente entre les informations complémentaires apportées par les différents types de données de notre corpus. D'autre part, face aux contraintes et spécificités du terrain qui ont émergé de façon progressive et évolutive, nous avons constamment réévalué nos problématiques et hypothèses de recherche, ainsi que nos stratégies d'approche et de positionnement, et adapté en conséquence les modalités d'observation initialement prévues, dans une dynamique d'influence et de modification réciproques qui a caractérisé le cheminement particulier de notre recherche. Suivant la même logique, le travail de recherche et d'ingénierie de la formation, se sont finalement imbriqués et rétro-alimentés.

Dans le cas spécifique du traitement analytique des diverses données collectées, nous avons suivi une stratégie de raffinement progressif d'hypothèses (Veillard [19] en référence à Engle et al., 2007 [24]). Elle nous a tout d'abord permis de réduire et essentialiser un corpus conséquent pour en déduire les caractéristiques pertinentes ayant trait aux difficultés et lacunes manifestées sur le terrain quant à la transmission des compétences langagières et interactionnelles sollicitée par l'activité de service en restauration. Nous avons identifié et décrit de façon la plus fine possible ces lacunes et difficultés, puis élaboré des hypothèses pour en expliquer l'origine et le fonctionnement. Nous nous sommes ensuite basée sur ces hypothèses pour proposer des solutions didactiques aux problèmes de transmission observés en tenant compte des leviers et facilitateurs potentiels rencontrés dans la formation, demeurant jusqu'alors trop cloisonnés ou sous-exploités pour bénéficier de façon efficace à l'enseignement-apprentissage des compétences visées. Ainsi, cette démarche nous a permis de mettre en place des lignes d'action concrète de remédiation à intégrer au cursus de formation selon nos objectifs de recherche. Finalement, Le résultat de notre analyse nous permet de constater que nos objectifs initiaux ont globalement été atteints, au vu de l'engagement des acteurs concernés 
(formateurs et étudiants), de leur investissement des outils proposés, et de la progression attestée des étudiants quant à l'apparition, au cours de l'activité de service en restaurants-écoles, d'une expertise professionnelle liée à la maîtrise des compétences langagières et interactionnelles.

$\mathrm{Au}$ regard de ce bilan général, nous souhaitons souligner les apports considérables, tant en termes de dynamique de recherche que d'ingénierie de la formation, que le choix de la démarche ethnographique et ses modalités particulièrement intéressantes de production de données ont permis de générer. Appliquées à un cas concret d'amélioration des pratiques et méthodes de formation au métier de service en restauration en faveur d'une revalorisation de la profession, ce processus de recherche nous a donné les moyens de proposer et mettre en œuvre de nouvelles modalités de transmission des compétences langagières et interactionnelles basées sur une dynamique collective de transfert de compétences. De même, il nous paraît important de mettre en avant la plus-value possible que la transmission-acquisition des compétences langagières et interactionnelles représente pour l'expertise professionnelle dans le secteur de la restauration, et de manière plus globale, dans le secteur du service.

\section{Bibliographie}

1. C. Hugol-Gential, Le service au restaurant: Analyse linguistique et multimodale des interactions entre personnel de service et clients, Thèse de Doctorat en Sciences du Langage, Laboratoire ICAR, Lyon 2 (2012)

2. P. Kunégel, Les maîtres d'apprentissage. Analyses des pratiques tutorales en situation de travail, L'Harmattan, Paris (2011)

3. P. Mayen, Travail de relation de service, compétences et formation, in Cerf, M. et Falzon, $\mathrm{P}$. (dir.), Travailler dans le service, pp. 59-81, Coll. Le travail humain, P.U.F., Paris (2005)

4. P. Pastré, P. Mayen, G. Vergnaud (2006). La didactique professionnelle. Revue française de pédagogie, 154, pp. 145-198 (2006)

5. S. Billett, Learning in the workplace : strategies for effective practice, Crows Nest : Allen et Unwin (2001)

6. J. Lave, E. Wenger, Situated Learning. Legitimate peripheral participation, Cambridge University Press, Cambridge, 138 p. (1991)

7. E. Goffman, Les rites d'interaction, Minuit, Paris (1967)

8. E. Goffman, La mise en scène de la vie quotidienne, Minuit, Paris (1973)

9. E. Goffman, Les Cadres de l'expérience, Minuit, Paris (1974)

10. J. Gumperz, Engager la conversation, introduction à la sociolinguistique interactionnelle, Minuit, Paris, (1989)

11. D. Hymes, Vers la compétence de communication, Didier, Paris (1984)

12. L. Mondada, Interactions en situations professionnelles et institutionnelles: de l'analyse détaillée aux retombées pratiques, Revue française de linguistique appliquée, XI(2), pp. 5-16 (2006)

13. J. Searle, Les actes de langage. Essai de philosophie linguistique, Hermann, Paris (1972)

14. L. Vygotsky, Pensée et Langage. La Dispute, Paris (1985)

15. L. Filliettaz, I. De Saint-Georges, B. Duc, "Vos mains sont intelligentes!": Interactions en formation professionnelle initiale, Cahiers de la section des sciences de l'éducation, 117, Université de Genève, Genève (2008)

16. G. Kress et al., Multimodal Teaching and Learning. The Rhetorics of the Science Classroom. Continuum, Londres et New York (2001)

17. A. Borzeix, B. Gardin (Eds), Langage et activité de service, Cahiers Langage et travail 4 (1992)

18. J. Boutet, La part langagière du travail. Bilan et évolution, In Langage et Société, 98, pp. 17-42 (2001)

19. L. Veillard, Les méthodologies de constitution et d'analyse des enregistrements données vidéo dans les recherches en éducation, dans L. Veillard et A. Tiberghien (Eds.), ViSA : Instrumentation de la recherche en éducation (2013) 
20. C. Lallier, Pour une anthropologie filmée des interactions sociales, Ed. des Archives contemporaines, Paris (2009)

21. J. Gumperz, D. Hymes (éds), The ethnography of communication, publication spéciale de l'American Anthropologist 66(6) (1964)

22. O. Baude et al., Corpus oraux, Guides des bonnes pratiques, CNRS-Editions et Presses Universitaires d'Orléans (2006)

23. J.-P. Olivier de Sardan, «La politique du terrain », Enquête, 1/1995, pp. 71-109 (1995)

24. R. A. Engle, F. R. Conant, J. G. Greeno, Progressive refinement of hypotheses in video-Supported Research, in R. Goldman et al. (dir.), Video Research in the Learning Sciences, Mahwah (NJ), Erlbaum : 239-254. (2007) 
